\title{
Mobility Mode Detection Using WiFi Signals*
}

\author{
Arash Kalatian $^{\dagger} \quad$ Bilal Farooq ${ }^{\ddagger}$
}

September 18, 2018

\begin{abstract}
We utilize Wi-Fi communications from smartphones to predict their mobility mode, i.e. walking, biking and driving. Wi-Fi sensors were deployed at four strategic locations in a closed loop on streets in downtown Toronto. Deep neural network (Multilayer Perceptron) along with three decision treebased classifiers (Decision Tree, Bagged Decision Tree and Random Forest) are developed. Results show that the best prediction accuracy is achieved by Multilayer Perceptron, with $86.52 \%$ correct predictions of mobility modes.
\end{abstract}

\footnotetext{
${ }^{*}$ Published in the proceedings of IEEE International Smart Cities Conference 2018

${ }^{\dagger}$ Laboratory of Innovations in Transportation (LiTrans), Ryerson University, Canada, Email: arash.kalatian@ryerson.ca

${ }^{\ddagger}$ Laboratory of Innovations in Transportation (LiTrans), Ryerson, Canada, Email: bilal.farooq@ryerson.ca
} 


\section{Introduction}

Conventionally, customized surveys have been conducted to gather required sample. Intrinsic problems of traditional methods include involvement of human errors and biased responses, as well as being timeconsuming, expensive and not representative. As a result, using location-aware technologies to collect data have gained popularity among scholars in different fields [Farooq et al., 2015]. These modern approaches in data collection have led to a dramatically growing interest in utilizing these data for mobility purposes.

Location aware technologies that have been implemented in mobility data collection processes include: body worn sensor, GPS data, GSM data, Wi-Fi data, Bluetooth transceivers data and RFID tags data. In general, these data collection approaches can be divided into two main categories: 1 . User Centric Approaches and 2. Network Centric Approaches. User Centric Approaches, such as GPS or body worn sensors, require users to be actively involved in data collection procedure. Turning on device's GPS and installing and running a mobile application to collect GPS records for instance, make it hard to use these data in real life mobility problems. In Network Centric Approaches, such as GSM, Wi-Fi or Video Recording methods on the other hand, data can be collected passively with no intervention from users.

Due to less infrastructure required, easier data preparation and calibration and more accuracy, GPS, GSM and Wi-Fi are the most referred source of data in related literature among other locationaware technologies: GPS-based approaches are the most precise sources of data in terms of geolocalization. The necessity of turning on mobile phones' GPS modules, however, makes these methods extremely battery draining. In addition, intervention from users is required (by turning on GPS modules or installing particular applications on their device), which prevents collecting data passively. Using cellular networks data resolves the problem of battery consumption and users intervention. Lack of accuracy in spotting user location, however, makes this source of data unreliable for estimating travel features in which accuracy plays an important role. By implementing sensors with the ability to collect connection information from Wi-Fi enabled devices, location and movement data of users can be inferred passively with no intervention from users Farooq et al., 2015]. Noted advantages of using Wi-Fi data led us to study the feasibility and accuracy of exploiting Wi-Fi data to detect mobility mode.

Inferring mode of mobility is of interest as it gives insight into shares of different modes and their changes and trends over time. This information can be used in urban planning, contextual advertisement and designing bike lanes or sidewalks. Being able to extract mobility mode information in short period of time can help city decision makers to better prepare city infrastructure based on modal share. Other applications of inferring mobility mode include, but not limited to, carbon footprints of users with different modes, daily step count of users and estimation of traffic and pedestrian flow.

Although studies have been conducted in this field, they have either focused on indoor environments, or have coupled Wi-Fi data with data from other sources. To the best of our knowledge, this is for the first time that mode detection is achieved using Wi-Fi as the only source of data on urban streets. Data used in this study are collected by URBANFlux system, consisting of OD_Pod detectors. These detectors are able to record signals emitted by Wi-Fi enabled smartphones carried by most networks users. URBANFlux system is described in detail in Farooq et al. 2015. For the purpose of this research, eight detectors were installed on specific parts of downtown Toronto streets to collect Wi-Fi tracks of the participants walking, biking, or driving. Data collected in our experiment are used to train classifiers to predict mobility mode based on a set of defined features. Decision Tree, Bagged Decision Tree, Random Forest and Multilayer Perceptrons are used to predict participants mobility modes.

The rest of the paper is organized as follows: next section reviews previous works on the subject. 
We then describe our data collection and extraction procedure in detail. In the methodology section, features are explained and the algorithm implemented for selecting the most important ones is discussed. Classification techniques used in this study and their comparative analysis are then elaborated on. In the end, conclusions and future research plans are outlined.

\section{Background}

The general approach for determining mobility mode using smartphones in the existing literature consists of: (a) extracting related features from raw sensor data, (b) training an algorithm and (c) predicting mobility mode for the unseen data. GPS sensors have been the main source of data in related works, alone or along with additional sources. Zheng et al. 2008] use GPS data solely to detect mode of mobility. Using the approach suggested in this study, mobility mode of users in $76 \%$ of the experiments are predicted correctly.

To add to the accuracy of the results, using multiple data sources has been widely practiced in related studies. Reddy et al. [2008], for instance, implement GPS data along with smartphones accelerometer data to distinguish users movements between walking, running, biking and motorized traveling. An accuracy of $93.6 \%$ is reached using two-stage decision tree and discrete Hidden Markov Model. In another study, Stenneth et al. 2011], add data from transportation network to determine users' mode of mobility between stationery, walking, biking, driving, and using public transit. Various methods including Decision Tree, Random Forest, Naïve Bayesian and Multilayer Perception are used in this study. Random Forest Method is chosen as the best classification method reaching an accuracy of $93.7 \%$. Dabiri and Heaslip 2018] utilized a convolutional neural network scheme to extract the mode of mobility using raw GPS data. They achieved an accuracy of $84 \%$ in detecting walking, driving, biking, using bus and train.

In spite of the high accuracy in GPS-based methods, requiring users intervention, high battery consumption and difficulties for wide-spread implementations of such methods, have led scholars to use other, although less accurate, sources. The basic idea behind users' geolocalization with GSM data is to acquire their location based on the learning of which Based Transceiver Stations (BTS) they are connected to in specific time spans. Sohn et al. [2006], for example, use coarse grained GSM data to determine whether a user is staying in a place, walking or driving. Boosted Logistic Regression is used in two phases and an accuracy of $85 \%$ is reached in this study. Low positioning accuracy, ping-pong handover effect and privacy concerns have been mentioned in this study as some of the main problems of using GSM data. In addition, relatively low density of BTS in some areas make GSM data an untrusted source for detecting mode specially at local levels. For trips within a block or neighborhood, for instance, GSM data cannot be used as a BTS cell size is at least 200 meters Kalatian and Shafahi, 2016.

Wi-Fi enabled devices can be discovered when they are inside Wireless Local Area Network. Being able to detect devices within access point's wireless range with no need of logging on, makes passive collection of data feasible. Mun et al. 2008 coupled Wi-Fi and GSM data to reach a classification accuracy of $87 \%$ in urban areas. Features used for classification in this experiment include: Wi-Fi signal strength Variance, duration of dominant Wi-Fi access point, number of cell IDs that device connects to and residence time in cell footprint. A decision tree is trained for the purpose of this study.

Considering the advantages of exploiting Wi-Fi data to detect mobility mode, along with the fact that past studies with same source of data have mainly focused on limited indoor movements, or have added data obtained from other sources, make us explore the feasibility of using W-Fi data as the only source of data to detect mobility mode. 


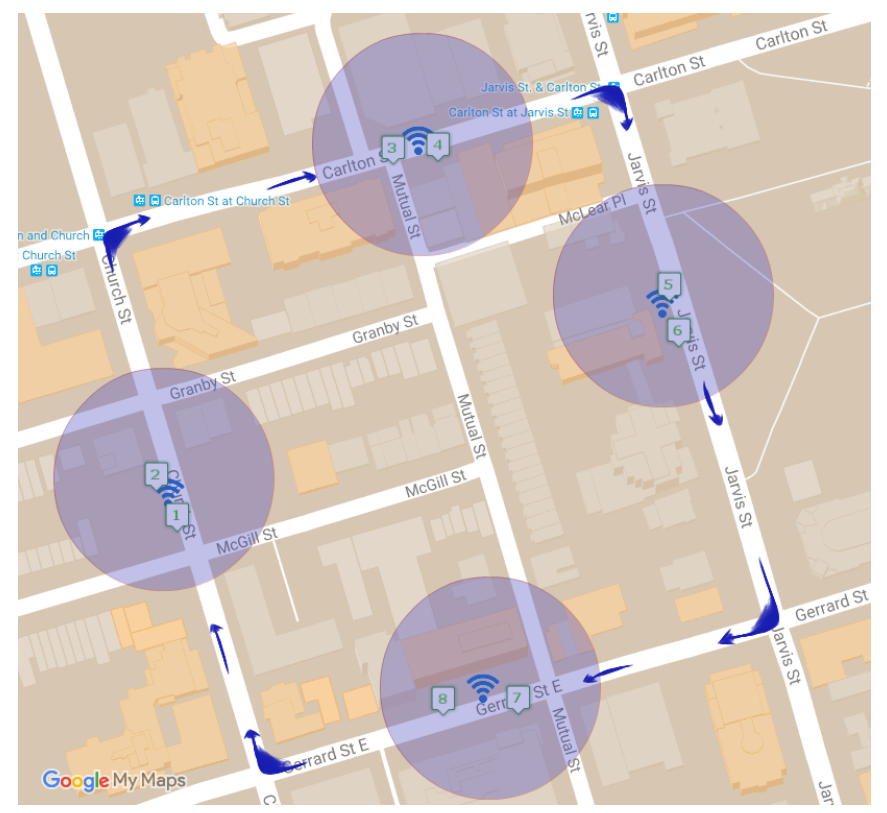

Figure 1: Experiment area and OD_Pods location

\section{Data}

Data used in this study is collected by URBANFlux system consisting of a network of detectors (named OD_Pods) as described in Farooq et al., 2015]. Every phone and computer has a unique hardware code, known as Media Access Control (MAC), by which it is identified. Wi-Fi enabled devices broadcast their MAC addresses periodically in order to explore available Wi-Fi Networks. URBANFlux detectors record these MAC addresses along with signal strengths and times of connection for individual devices carried by users who are in their coverage zone. Coverage zone of these detectors can be approximated as an sphere with a radius of 50 meters. Therefore, it is assumed in this study that connection details of mobile phones are recorded by an OD_Pod when their direct distance to that OD_Pod is less than 50 meters.

Parts of four downtown Toronto streets forming a loop were selected for installing OD_Pods. This area was selected in order for the experiment to be as realistic as possible. Data collection is conducted from 10 A.M. to 1 P.M on a weekday so that traffic on the streets resemble regular everyday life conditions. As it is depicted in Figure 1, the selected parts form a grid loop with a perimeter of 857 meters. Coverage areas for OD_Pods and direction of participants moving are also depicted in Figure1. Two OD_Pods were installed in each location to decrease the chances of missing records due to possible OD_Pods problems. To avoid signal overlaps, mid block points were chosen to install OD_Pods.

In this study, four lab members from LITrans were recruited for data collection procedure, each going around the designated loop for 10 rounds. Participants used different modes of mobility in a way that approximately equal number of data are recorded for each mode. Data recorded from movements between each two OD_Pods are considered as a trip with their respective mode. As the data used in the analysis was related to recruited participants, ground truth modes of transport were known.

By installing eight OD_Pods at mid-block locations, travel times can be measured for participants. Data are collected from participants with different modes of mobility: walking, biking and driving. Having connection time and distance between OD_Pods, a relative travel speed for participants is calculated as the normalized ratio of distance and time. Relative speed along with features such as 
Table 1: Total number of trips collected for each mode

\begin{tabular}{lc}
\hline Mode & Number of Trips \\
\hline \hline Walking & 142 \\
Biking & 108 \\
Driving & 150 \\
\hline
\end{tabular}

average and variance of signal strengths and other derived features are then used to infer mode of mobility of participants. The details of feature extraction and classification are further elaborated in next section.

\section{Methodology}

Intuitively, mobility patterns for different mobility modes are different. Users' modes can be classified based on these differences in the patterns. In regard to algorithm performance, previous studies have suggested that the Decision Tree-based approaches perform well in terms of classifying these patterns. In addition, Deep Neural Networks (DNN) have been widely used and have performed successfully in various fields in recent years. In this section, we first discuss the importance of feature selection and variable importance ranking in classification. We then describe "ReliefF algorithm" implemented in this study for feature selection. Next, decision tree-based algorithms and DNN algorithm used for predicting mobility mode are discussed in detail.

\subsection{ReliefF algorithm}

A feature selection algorithm should be utilized before using dependent variables as inputs of any machine learning algorithm for classification. The main reason for determining variable importance before training a classifier is making the calibration process of base learners more efficient. In this study, ReliefF algorithm is used to rank variables based on their importance. ReliefF algorithm was proposed by Kononenko et al. [1997] for multinomial classification. The basic idea of Relief Algorithms family is to estimate the quality of variables based on their ability to distinguish between observations that are close to each other.

\subsection{Decision Tree}

Decision Tree (DT) classification uses a decision tree structure to predict class of observations based on their features. Basically, a tree is learned by testing attribute values, and splitting the source set to subsets based on that. For every derived subset, this procedure is repeated until no improvement is observed on predictions. The core algorithm for building decision trees employs a top-down, greedy search through the space of possible branches. Details of DT algorithm for classification can be found in the work of Breiman et al. 1984.

\subsection{Bagged Decision Tree}

To improve prediction accuracy of DT, ensemble learning algorithms are practiced. Fundamentally, an ensemble learning algorithm is using combined results of a set of classifiers. In this study, Bagged Decision Tree (BDT) is used to reduce the error of prediction for individual decision trees. In BDT, a number of Decision Trees are grown with samples from training data set. Samples are drawn from training set randomly and with replacement. Number of Decision Trees $(n)$ should be optimally tuned. 
Error is reduced with increasing the number of trees, and remains stationary after an optimal value of $n$.

Algorithm 1 describes the bagging algorithm used in BDT. Sample sizes are equal to the size of training dataset, so in bagging training set, some observations may appear several times. Each tree is calibrated individually and the predictions are combined by considering majority vote of trees. The idea behind growing multiple trees is that diversity among DT classifiers, compensates for the increase in error rate of each individual classifier.

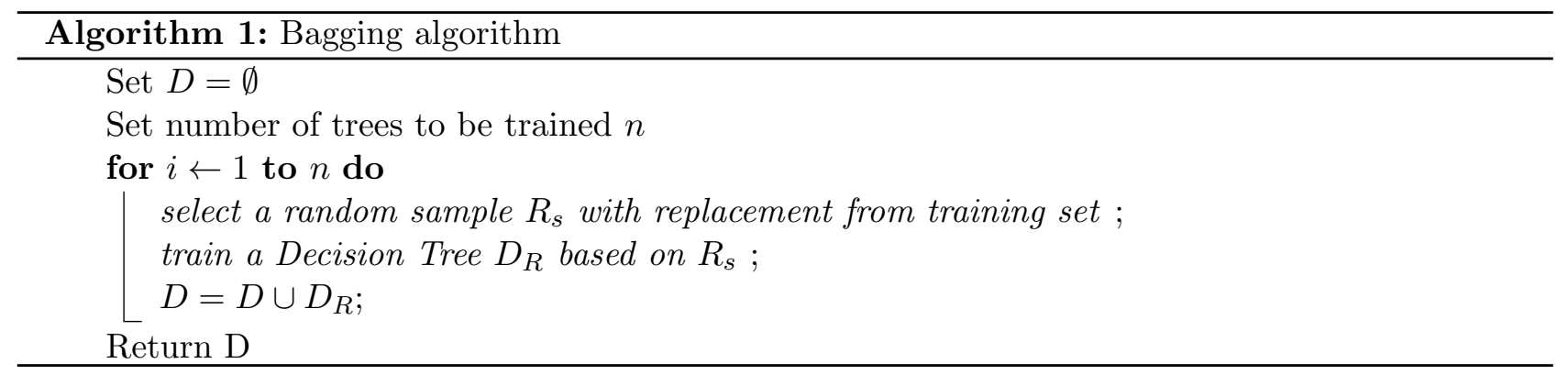

\subsection{Random Forest}

Another ensemble Decision Tree-based algorithm is Random Forest (RF), developed by Breiman 2001. In RF, in addition to bootstrap sampling of observations explained in BDT section, a subset of input variables are randomly selected as features for each tree. The pseudopod for RF algorithm is presented in Algorithm 2 Liaw et al. 2002. To predict the class of new data, predictions from $n_{t}$ trees are

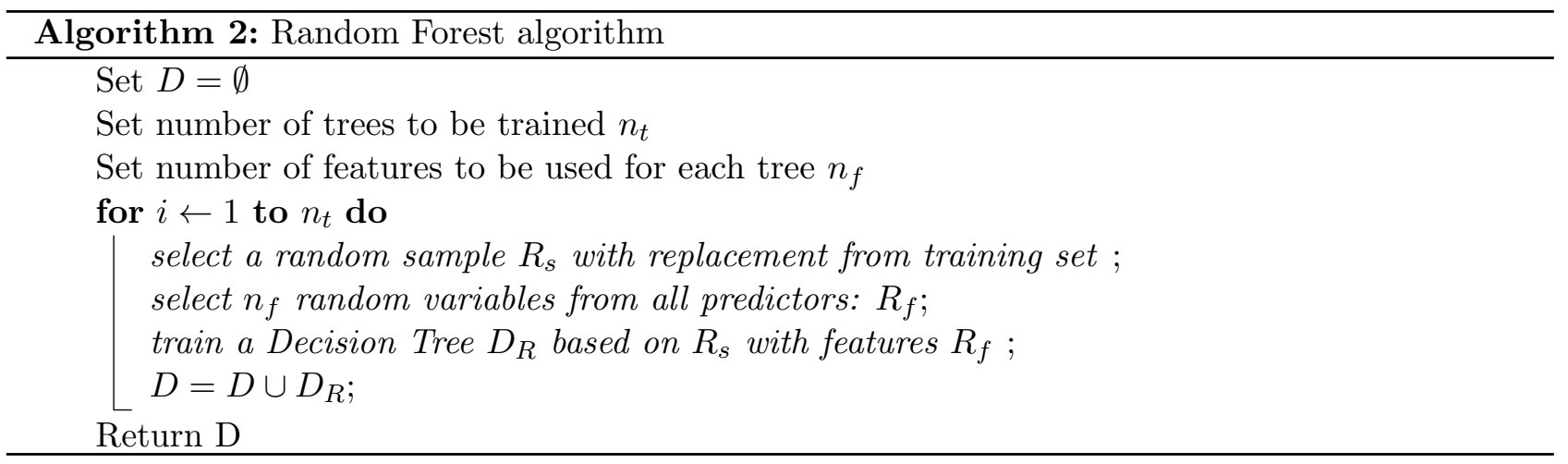

aggregated by considering majority vote. As it is shown in Algorithm 2, two parameters should be optimally tuned: number of trees $\left(n_{t}\right)$ and number of features $\left(n_{f}\right)$.

\subsection{Multi-layer Perceptron}

Introduced in Rumelhart et al. [1986], Multilayer Perceptron (MLP) is a feed-forward artificial neural network. MLP consists of an input layer on one end, an output layer on the other end and an arbitrary number of hidden layers in between. The information start in the input layer and propagate through the network layer by layer (Honkela 2001). In the mathematical form, each Perceptron can be written as in equation 1 .

$$
y=\varphi *\left(W^{T} * x+b\right)
$$


where $\varphi$ is the activation function, $\mathrm{W}$ is the vector of weights, $\mathrm{b}$ is the bias and $\mathrm{x}$ is the vector of inputs.

The perceptron forms a linear combination of inputs and their weights to compute an output, and puts the output in a nonlinear activation function. The perceptrons will then be used as parts of a more complex structure in MLP. In the MLP architecture, number of nodes in input layer and output layer represent number of features and number of labels (modes in our study) respectively.

MLPs have been used successfully in different areas, such as speech recognition, image recognition, and machine translation. For the purpose of this study, we utilize a MLP scheme for mode detection using Keras Package in R [Chollet et al., 2017].

\section{Results}

\section{$5.1 \quad$ Features}

Having MAC addresses of participants' devices, connection data belonging to each participant can be separated. Every movement between two OD_Pods is considered as a trip observation, which includes data from origin OD_Pod and destination OD_Pod. Based on connection details from origin and destination OD_Pods, 15 variables are extracted as possible features for classification. These variables can be categorized in 4 groups in general, which are:

1. Time Related Variables: Relative Travel Speed and connection time variables are included in this group. Relative Travel Speed is represented by the normalized ratio of "distance with no coverage zone between two involved OD_Pods", to its respective travel time. Speed related variables have been the main feature for classification in related literatures. However, using speed solely does not guarantee satisfactory results. This can be explained by the fact that in congested areas, different mobility modes move with similar speeds.

2. Connection time variables represent variables related to the time that a device is discovered in the coverage zone of an OD_Pod.

3. Connection Number Variables: number of connections to an OD_Pod while a device is in its coverage zone. Intuitively, when a user spends more time in a coverage zone, for example walks in a coverage zone, number of connections increases.

4. Signal Strength Variables: Variance, first and second derivative of signal strengths during the time a device is connected to an OD_Pod.

\subsection{Variable Importance}

Using ReliefF algorithm, a ranking of variables based on their weighted importance is obtained. Variables with their respective units, ranks and weight importances are presented In Table 2, As it can be inferred from the table, variables regarding number of connections are the most relevant features for determining mobility mode. When participants move with a slower speed, their device spends more time in a coverage zone, resulting in more number of connections and time. As expected, relative speed is another key variable for training a classifier. As it is presented in Table 2, all the weights calculated have positive values, which means all of them should be taken into account for classification. 
Table 2: Details of features defined for classification and their weight importance and ranking

\begin{tabular}{|c|c|c|c|c|}
\hline ID & Variable & Unit & Rank & Weight \\
\hline \multicolumn{5}{|c|}{ Time } \\
\hline 1 & Relative Travel Speed & - & 4 & 0.050 \\
\hline 2 & Origin Connection Time & $\mathrm{s}$ & 7 & 0.033 \\
\hline 3 & Destination Connection Time & $\mathrm{s}$ & 9 & 0.020 \\
\hline \multicolumn{5}{|c|}{ Connection Number } \\
\hline 4 & Number of Connections-Orig & - & 2 & 0.073 \\
\hline 5 & Number of Connections-Dest & - & 3 & 0.059 \\
\hline 6 & Number of Connections-Avg & - & 1 & 0.104 \\
\hline \multicolumn{5}{|c|}{ Signal Strength } \\
\hline 7 & Signal Strength Variance-Orig & $d B m^{2}$ & 11 & 0.010 \\
\hline 8 & Signal Strength Variance-Dest & $d B m^{2}$ & 10 & 0.010 \\
\hline 9 & Signal Strength Variance-Avg & $d B m^{2}$ & 6 & 0.036 \\
\hline 10 & Signal Strength 1st Derivative-Orig & $\mathrm{dBm} / \mathrm{s}$ & 5 & 0.036 \\
\hline 11 & Signal Strength 1st Derivative-Dest & $\mathrm{dBm} / \mathrm{s}$ & 14 & 0.003 \\
\hline 12 & Signal Strength 1st Derivative-Avg & $\mathrm{dBm} / \mathrm{s}$ & 8 & 0.021 \\
\hline 13 & Signal Strength 2nd Derivative-Orig & $d B m / s^{2}$ & 13 & 0.006 \\
\hline 14 & Signal Strength 2nd Derivative-Dest & $d B m / s^{2}$ & 15 & 0.0002 \\
\hline 15 & Signal Strength 2nd Derivative-Avg & $d B m / s^{2}$ & 12 & 0.0009 \\
\hline
\end{tabular}

\subsection{Classification}

Classification process starts with training a DT. In spite of easy calibration process, DT does not result in satisfying accuracy. The minimum number of leaf node observations and branch node observations are set to their default value, 1 and 10 respectively. Confusion matrix for test data for DT is presented in Table 3. In this table, actual number of trips is compared to DT predictions for different modes of travel. Column Recall for each mode indicates the number of correct predictions for that mode divided by total number of trips with that mode. In row Precision, number of correct predictions for each mode is divided by total number of times that a mode is predicted. As it is provided in Table 3 , biking is the least accurately predicted mode with a recall of $47.91 \%$ and precision of $60.52 \%$. Total number of correct predictions in DT appears to be $68.75 \%$.

To reduce the errors of DT and improve the classification prediction accuracy, BDT algorithm is implemented. To use BDT for classification, number of trees included in BDT, $n$, should be calibrated. Different numbers of trees are tested in this study to calculate the optimal value of $n$. The value of $n$ is set to be $10,20,50,100,200,500,1000$, and it turned out that the prediction accuracy remains approximately constant for $n$ grater than 200. Confusion matrix of BDT for test data is provided in Table 4. As the recall and precision values of this table suggest, implementing bagging strategy improves recall and precision for all modes, except a decrease in recall percentage of walking. In addition, the total prediction accuracy increases from $68.75 \%$ for DT to $78.25 \%$ in BDT.

As the last DT based algorithm, mobility mode is predicted by training a RF algorithm. For RF, the recommended value of $n_{f}$ is one third of total number of predictors [Saadi et al. 2017, which means setting $n_{f}=5$. To ensure about the optimum values of $n_{t}$ and $n_{f}$, multiple numbers are set and tested for both parameters. Predictions of RF trained with different parameter values appears to be most accurate with $n_{t}=400$ and $n_{f}=5$, which confirms the recommendation of setting number of randomly selected predictors equal to one third of total number of features. The Confusion Matrix of $\mathrm{RF}$ for test data is presented in Table 5. A total prediction accuracy of $83.13 \%$ is reached using $\mathrm{RF}$ 
Table 3: Confusion Matrix for Decision Tree

Prediction

\begin{tabular}{|c|c|c|c|c|c|}
\hline \multirow[b]{2}{*}{ Walking } & Walking & Biking & Driving & \multirow[b]{2}{*}{$\begin{array}{c}\text { Total } \\
57\end{array}$} & \multirow[b]{2}{*}{$\begin{array}{c}\text { Recall\% } \\
92.98\end{array}$} \\
\hline & 53 & 3 & 1 & & \\
\hline Biking & 12 & 23 & 13 & 48 & 47.91 \\
\hline Driving & 9 & 12 & 34 & 55 & 61.81 \\
\hline To1 & tal 74 & 38 & 48 & 160 & \\
\hline Precisio & n 71.62 & 60.52 & 70.83 & & \\
\hline
\end{tabular}

Table 4: Confusion Matrix for Bagged Decision Tree

Prediction

\begin{tabular}{|c|c|c|c|c|c|}
\hline \multirow[b]{2}{*}{ Walking } & Walking & Biking & Driving & \multirow[b]{2}{*}{$\begin{array}{c}\text { Total } \\
57\end{array}$} & \multirow[b]{2}{*}{$\begin{array}{c}\text { Recall\% } \\
87.72\end{array}$} \\
\hline & 50 & 5 & 2 & & \\
\hline Biking & 4 & 36 & 8 & 48 & 75.00 \\
\hline Driving & 9 & 7 & 39 & 55 & 70.91 \\
\hline \multicolumn{2}{|c|}{ Total 63} & 48 & 49 & 160 & \\
\hline Precisiol & A 79.37 & 75.00 & 79.59 & & \\
\hline
\end{tabular}


Table 5: Confusion Matrix for Random Forest

Prediction

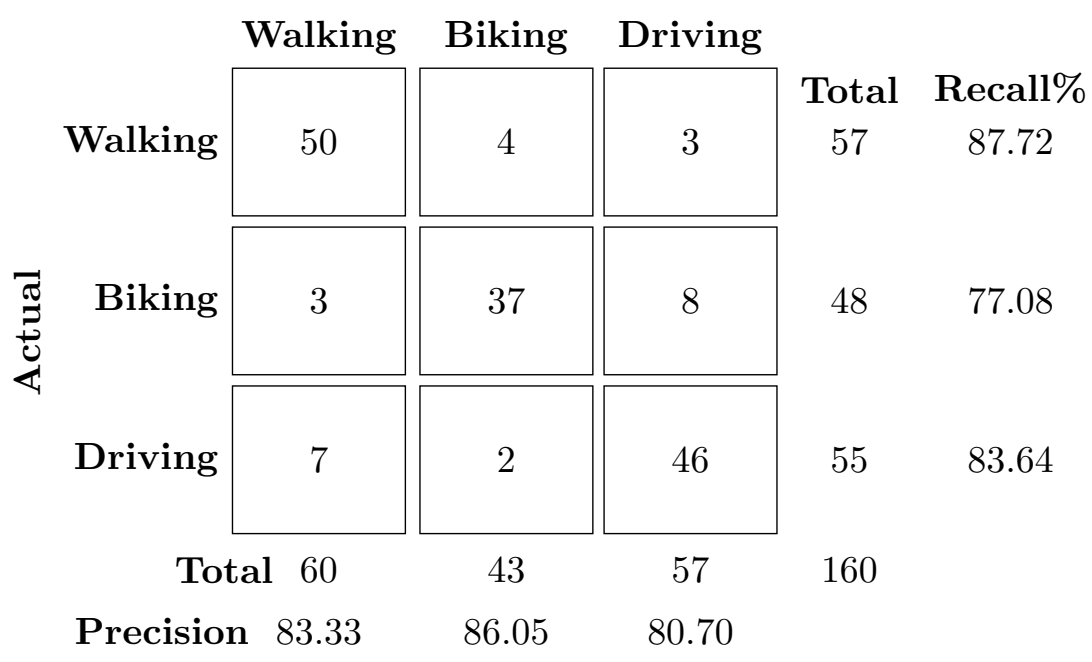

Table 6: Values for MLP parameters

\begin{tabular}{|l|l|}
\hline Name & Value \\
\hline Number of Epochs & 200 \\
\hline Optimization Method & ADAM \\
\hline Number of hidden layers & 4 \\
\hline Input and hidden layers & activation: RELU \\
\hline Number of nodes & all hidden layers: 15 \\
\hline Output layer & activation: softmax \\
\hline Batch size & 20 \\
\hline
\end{tabular}

algorithm. As it is presented in Table 5, recall and precision values for all modes is also improved, compared to results from BDT. Similar to previous methods, walking has the best recall percentage: 50 observations out of total 57 walking observations are predicted correctly. On the other hand, 10 biking or driving labeled observations are predicted as walking by mistake, which makes a precision of $83.33 \%$. This precision is still more precise than the results of DT and BDT. Both recall and precision for driving labeled observations are above $80 \%$, and greater than their respective values in two previous methods.

In the end, Multilayer Perceptron is used to predict mode of mobility. With multiple parameters to be tuned, it is more difficult to find an optimum MLP algorithm compared to DT based algorithm. These parameters include: Number of hidden layers, Number of nodes in each hidden layer, Optimization method, batch size and number of epochs. After implementing different architectures for the network, 87 percent accuracy in total predictions is achieved. Table 6 presents a summary of the final model attributes. As presented in this table, the number of epochs is set to be 200. An epoch is one complete presentation of the dataset to the learning algorithm. This number is set based on the accuracy of model is predicting validation and training datasets. The accuracy of the predictions for different number of epochs is presented in Figure 2. As it can be seen in this figure, No observable change occurs after 200 epochs. The confusion matrix for MLP algorithm is provided in Table 7. As it can be inferred from the table, comparing to all previous DT based algorithms, Recall and Precision have increased significantly. Driving remains the least accurate mode, with around 15 percent of driving mode predicted as other modes. This error could mainly be a result of congested traffic 


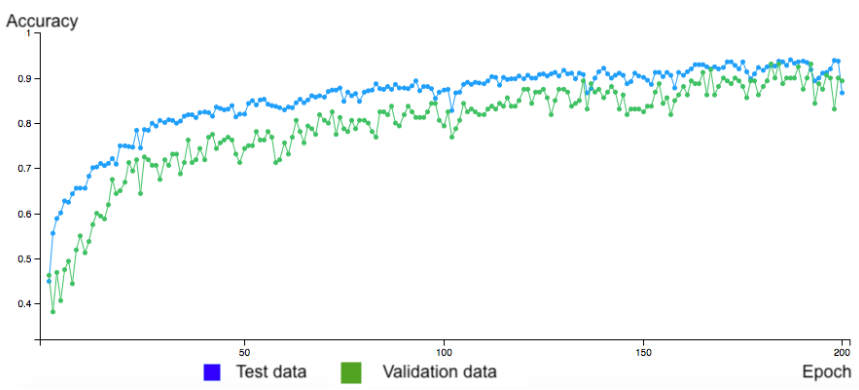

Figure 2: Accuracy of Training and Validation data on different epochs

Table 7: Confusion Matrix for Multilayer Perceptrons

\section{Prediction}

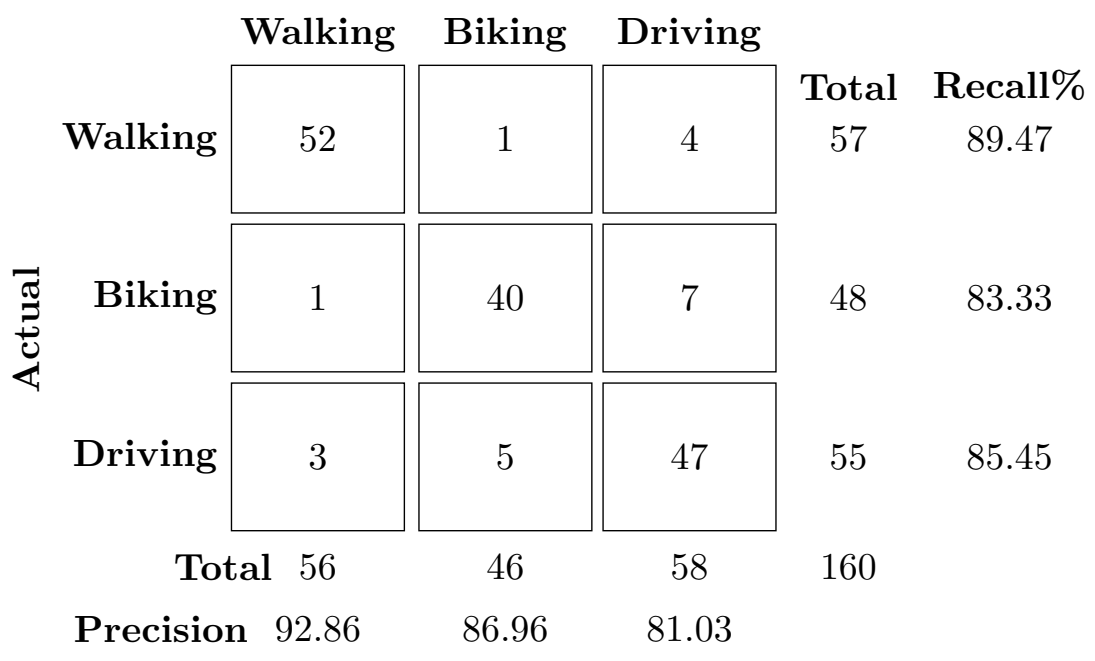

conditions at the intersections, which may make it hard to distinguish between different modes. Comparing to Random Forest algorithm, the total accuracy improved by 3 percent, which may be further be improved by using other deep learning algorithms. Finally, biking has the least recall percentage, mainly due to large number of biking observations predicted as driving. This error probably reflects the fact that in a congested area such that the data collection of this study was conducted, bikes and cars may share speed related features.

\section{Conclusions}

In this paper, we used Wi-Fi communication data for determining individual's mobility mode. URBANFlux technology Farooq et al., 2015] is used in this study to record MAC addresses along with signal strengths and time-stamp of communication from Wi-Fi enabled smartphones. Eight detectors were installed in four different locations in a designated loop route in downtown Toronto and four participants walked, biked and drove this route. Each movement between two OD_Pods in different locations is considered as an observation in the dataset, leading to a total of 400 observations.

Fifteen features are derived based on times and speeds, signal strengths and number of connections of observations. ReliefF algorithm is used to estimate the quality of predictors for feature selection. Three decision tree-based algorithms and one Deep Neural Network algorithm classifiers are trained and travel modes for test data are predicted. Calibrating and training a Multilayer Perceptron algo- 
rithm resulted in an accuracy of $86.52 \%$ in mode classification: $89.47 \%$ for walking, $83.33 \%$ for biking and $85.45 \%$ for driving mode.

Unlike other related works on this topic, in this study Wi-Fi data are not coupled with other sources of data to detect mobility mode in urban areas. This makes our proposed approach more cost-effective and easier to implement-with no interventions from users required. As long as smartphone Wi-Fi is on, which is almost always the case, we are able to detect users carrying them in the proximity of our detectors. Another key differentiating feature in this study is the implementation of Wi-Fi based mode detection on actual urban roads with real traffic at reasonably large scale. Regarding the accuracy in prediction, our study performs well comparing to other studies using solely Wi-Fi data. The highest accuracy in related studies can be obtained by This method can also be used within URBANFlux system by city decision makers and planners to have a better understanding of users travel habits and their trends over time. City of Toronto, for instance, is collecting and publishing travel times, counts and travel speeds data of vehicles from Bluetooth and WiFi sensors on streets and highways across the city (2014-2017). The same infrastructure can be simply improved to incorporate mode detection algorithms for estimation of modal in different parts of the city, with no additional burden on the city's economy. This method can also be used in a real-time within URBANFlux system to acquire information like traffic conditions or level of service for bike lanes and sidewalks.

In the context of future work, this study can be improved in different aspects. In terms of methodology, other feature selection algorithms and classification methods can be explored to compare the results with the current study. Taking heterogeneity of smartphones into account may also add to the precision of this study. Other travel modes, such as buses, subways or stationary mode can be investigated in future work. Considering the large unlabeled dataset that has been collected during this study, the models can further be improved using semi-supervised learning algorithms. In addition, dataset can be expanded to consist different parts of city, with more participants recruited to have a bigger

and more comprehensive dataset. URBANFlux technology allows affordable longterm monitoring of network users [Beaulieu and Farooq, 2017], which provides perspective on exploring other traffic characteristics such as routes, origin-destination tables, activity patterns, and fundamental relationships between speed, density, and flow of traffic.

\section{References}

Alexandra Beaulieu and Bilal Farooq. Large-scale pedestrian movement analysis using a network of wi-fi sensors. In ITS World Congress 2017: Integrated Mobility Driving Smart Cities, 2017.

Leo Breiman. Random forests. Machine learning, 45(1):5-32, 2001.

Leo Breiman, Jerome H Friedman, Richard A Olshen, and Charles J Stone. Classification and regression trees. 1984.

François Chollet, JJ Allaire, et al. R interface to keras. https://github.com/rstudio/keras, 2017.

Sina Dabiri and Kevin Heaslip. Inferring transportation modes from gps trajectories using a convolutional neural network. Transportation Research Part C: Emerging Technologies, 86:360-371, 2018 .

Bilal Farooq, Alexandra Beaulieu, Marwan Ragab, and Viet Dang Ba. Ubiquitous monitoring of pedestrian dynamics: Exploring wireless ad hoc network of multi-sensor technologies. In SENSORS, 2015 IEEE, pages 1-4. IEEE, 2015.

Antti Honkela. Nonlinear switching state-space models. Master's thesis, Helsinki University of Technology, Espoo, Finland, 2001. 
Arash Kalatian and Yousef Shafahi. Travel mode detection exploiting cellular network data. In MATEC Web of Conferences, volume 81, page 03008. EDP Sciences, 2016.

Igor Kononenko, Edvard Šimec, and Marko Robnik-Šikonja. Overcoming the myopia of inductive learning algorithms with relieff. Applied Intelligence, 7(1):39-55, 1997.

Andy Liaw, Matthew Wiener, et al. Classification and regression by randomforest. $R$ news, 2(3): $18-22,2002$.

M Mun, Deborah Estrin, Jeff Burke, and Mark Hansen. Parsimonious mobility classification using gsm and wifi traces. In Proceedings of the Fifth Workshop on Embedded Networked Sensors (HotEmNets), 2008.

The City of Toronto. Travel times - bluetooth, retrieved from https://www.toronto.ca/citygovernment/data-research-maps/open-data/, 2014-2017.

Sasank Reddy, Jeff Burke, Deborah Estrin, Mark Hansen, and Mani Srivastava. Determining transportation mode on mobile phones. In Wearable computers, 2008. ISWC 2008. 12th IEEE International symposium on, pages 25-28. IEEE, 2008.

David E Rumelhart, Geoffrey E Hinton, James L McClelland, et al. A general framework for parallel distributed processing. Parallel distributed processing: Explorations in the microstructure of cognition, 1:45-76, 1986.

Ismaïl Saadi, Melvin Wong, Bilal Farooq, Jacques Teller, and Mario Cools. An investigation into machine learning approaches for forecasting spatio-temporal demand in ride-hailing service. arXiv preprint arXiv:1703.02433, 2017.

Timothy Sohn, Alex Varshavsky, Anthony LaMarca, Mike Chen, Tanzeem Choudhury, Ian Smith, Sunny Consolvo, Jeffrey Hightower, William Griswold, and Eyal De Lara. Mobility detection using everyday gsm traces. UbiComp 2006: Ubiquitous Computing, pages 212-224, 2006.

Leon Stenneth, Ouri Wolfson, Philip S Yu, and Bo Xu. Transportation mode detection using mobile phones and gis information. In Proceedings of the 19th ACM SIGSPATIAL International Conference on Advances in Geographic Information Systems, pages 54-63. ACM, 2011.

Yu Zheng, Quannan Li, Yukun Chen, Xing Xie, and Wei-Ying Ma. Understanding mobility based on gps data. In Proceedings of the 10th international conference on Ubiquitous computing, pages 312-321. ACM, 2008. 